\title{
Making systems of Super Earths by inward migration of planetary embryos
}

\author{
Christophe Cossou ${ }^{1,2}$, Sean N. Raymond ${ }^{1,2}$ and Arnaud Pierens ${ }^{1,2}$ \\ ${ }^{1}$ Univ. Bordeaux, LAB, UMR 5804, F-33270, Floirac, France. \\ ${ }^{2}$ CNRS, LAB, UMR 5804, F-33270, Floirac, France
}

\begin{abstract}
Using N-body simulations with planet-disk interactions, we present a mechanism capable of forming compact systems of hot super Earths such as Kepler 11. Recent studies show that outward migration is common in the inner parts of radiative disks. However we show that two processes naturally tip the balance in favor of inward migration. First the corotation torque is too weak to generate outward migration for planetary embryos less massive than $4 \mathrm{M}_{\oplus}$. Second, system of multiple embryos generate sustained non-zero eccentricities that damp the corotation torque and again favor inward migration. Migration and accretion of planetary embryos in realistic disks naturally produce super Earths in resonant chains near the disk inner edge. Their compact configuration is similar to the observed systems.
\end{abstract}

Keywords. Planets and satellites: formation, Protoplanetary disks, Planet-disk interactions, planetary systems, Methods: numerical

\section{Introduction}

Super Earths $\left(1-10 \mathrm{M}_{\oplus}\right)$ are common (Mayor et al. 2011; Howard et al. 2012) even if none exist in our own solar system. They tend to be found in systems of multiple Super Earths in close-in, compact orbital configurations. For example, the Kepler 11 system contains five planets within the orbit of Mercury, each more massive than Earth (Lissauer et al. 2011).

Here we show how that gas disk-driven orbital migration naturally produces systems similar to the observed ones. We use N-body simulations that include torques from a 1-D gaseous disk to show how a system of planetary embryos starting at several AU embedded naturally migrates inward and accretes into a compact system of hot Super Earths. Some embryos can also grow and remain trapped on more distant orbits and presumably become giant planet cores.

\section{Methods}

We start from a 1D protoplanetary disk model, with parameters listed in Table 1. The key parameter is the surface density profile, defined as a power law in radial range $0.1<R<100 \mathrm{AU}$ :

$$
\Sigma(R)=\Sigma_{0}\left(\frac{R}{1 \mathrm{AU}}\right)^{-d}
$$

The inner edge of the disk is smoothed with a tanh function on a length scale of $H\left(R_{\text {in }}\right)$, where $H$ is the scaleheight of the disk, so the density goes to zero at the inner edge. From the surface density profile, we self-consistently compute the temperature $T$, thermal diffusivity $\chi$, scaleheight $H$ and optical depth profiles $\tau$. To calculate the temperature 
we use the following energy equation :

$$
0=-C_{\mathrm{BB}}+H_{\mathrm{en}}+H_{\mathrm{irr}}+H_{\mathrm{vis}}
$$

with

$$
\begin{array}{rlrl}
C_{\mathrm{BB}} & =2 \sigma \frac{T^{4}}{\frac{3}{8} \tau+\frac{\sqrt{3}}{4}+\frac{1}{4 \tau}} & H_{\mathrm{en}} & =2 \sigma T_{\mathrm{en}}{ }^{4} \\
H_{\mathrm{irr}} & =2 \sigma T_{\star}{ }^{4} \frac{R_{\star}^{2}}{r^{2}}(1-\varepsilon) *\left[0.4 \frac{R_{\star}}{r}+r \frac{\mathrm{d}}{\mathrm{d} r}\left(\frac{H}{r}\right)\right] & H_{\mathrm{vis}}=\frac{9}{4} \nu \Sigma \Omega^{2}
\end{array}
$$

$C_{\mathrm{BB}}$ is the disk's black body cooling and $H_{\mathrm{en}}$ is the black body heating by the disk envelope. $H_{\mathrm{vis}}$ is the viscous heating. $H_{\mathrm{irr}}$ is the heating from stellar irradiation (Chiang \& Goldreich 1997; Menou \& Goodman 2004), $\sigma$ the Stephan-Boltzmann constant, $\nu$ the viscosity of the disk, $T_{\star}$ and $R_{\star}$ the temperature and radius of the central star respectively, $\Omega$ the angular speed in the disk at a given position and $\varepsilon$ the disk's albedo. Starting at the outer edge of the disk, where we impose that $T=10 \mathrm{~K}$, we retrieve the temperature profile by solving eq. (2.2) numerically.

We use the formulae of Paardekooper et al. (2011) to compute the torque exerted by our 1D disk on a planet of a given mass at a given orbital radius. The main differences with the model described in this paper are :

- The temperature profile is not a power law in our model, but instead a local power law between each point of a table of several hundred points spaced in orbital distance

- the scaleheight of the disk, defined as $H=\frac{1}{\Omega} \sqrt{\frac{k_{B} T}{\mu m_{H}}}$, is computed following the temperature profile, with $k_{B}$ the Boltzmann constant, $\mu$ the mean molecular weight and $m_{H}$ the mass of an hydrogen atom.

We implement type I eccentricity and inclination damping following Cresswell \& Nelson (2008). We also include an eccentricity-migration feedback whereby the corotation torque is weakened for eccentric orbits (Bitsch \& Kley 2010), using the equations from (Cossou et al. 2013).

$$
\begin{aligned}
& \hline|b / h=0.6| \gamma=7 / 5|\mu=2.35| \alpha=5 \cdot 10^{-3}\left|T_{\star}=5700 \mathrm{~K}\right| R_{\star}=4.65 \cdot 10^{-3} \mathrm{AU} \mid \\
& \hline \text { Disk Albedo }=0.5 \mid \text { Disk }: \mathrm{R} \in[0.1 ; 100] \mathrm{AU} \mid \quad \Sigma(R)=300 \cdot R^{-1 / 2} \mathrm{~g} / \mathrm{cm}^{2} \\
& \hline
\end{aligned}
$$

Table 1. Parameters of the disk. In addition to those parameters, note that the opacities were retrieved from the opacity table of Huré (2000). The viscosity is modeled via the $\alpha$-prescription (Shakura \& Sunyaev 1973).

Disk forces were added to the hybrid version of the Mercury integrator (Chambers 1999). Drag and migration forces are not applied to objects inside the inner cavity (inside $0.1 \mathrm{AU})$. Collisions were treated as inelastic mergers.

\section{Mechanism}

The right panel of Figure 1 shows the disk's migration map. Two zones of convergent migration exist. The first convergence zone causes all embryos between 0.2 and $0.8 \mathrm{AU}$ to migrate toward $0.5 \mathrm{AU}$. The second causes embryos between 0.9 and $\sim 100$ AU to migrate toward $\sim 15$ AU. However, multiple planet systems do not actually migrate to convergence zones like isolated planets. Rather, they become trapped in chains of mean motion resonances. The resonant configurations sustain eccentricities large enough to attenuate the corotation torque (Bitsch \& Kley 2010). The system stabilizes in an equilibrium 

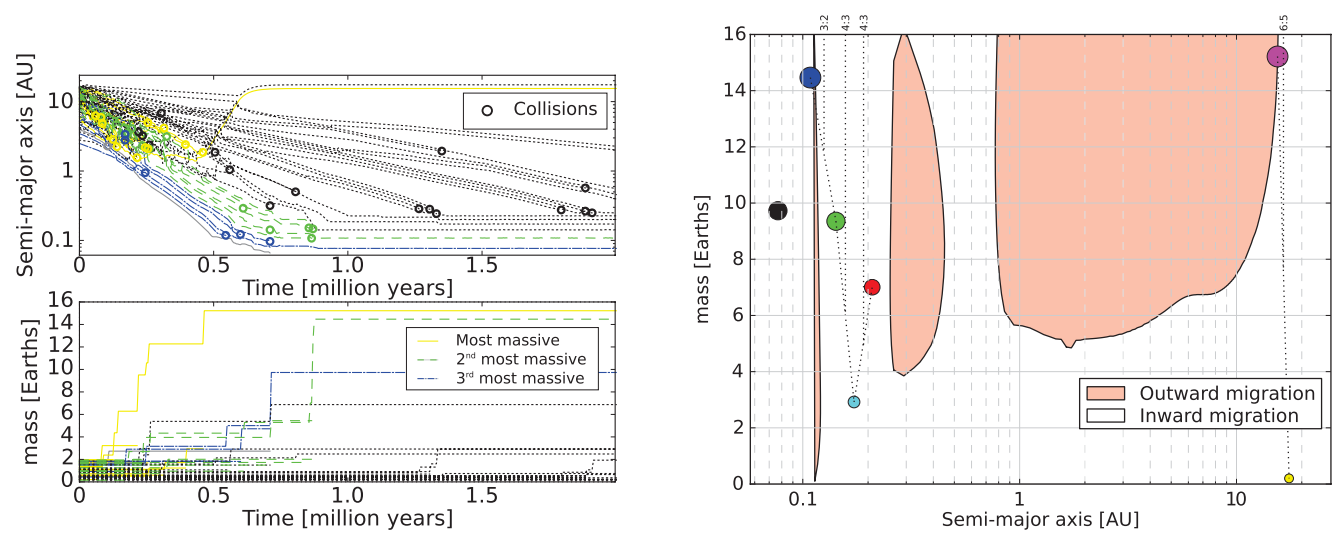

Figure 1. A simulation that forms a compact system of hot Super Earths. Left: The orbital evolution (top) and mass growth (bottom) of the embryos. Right: The final state of the system. Black lines represent zero-torque zones where isolated planets should stop migrating.

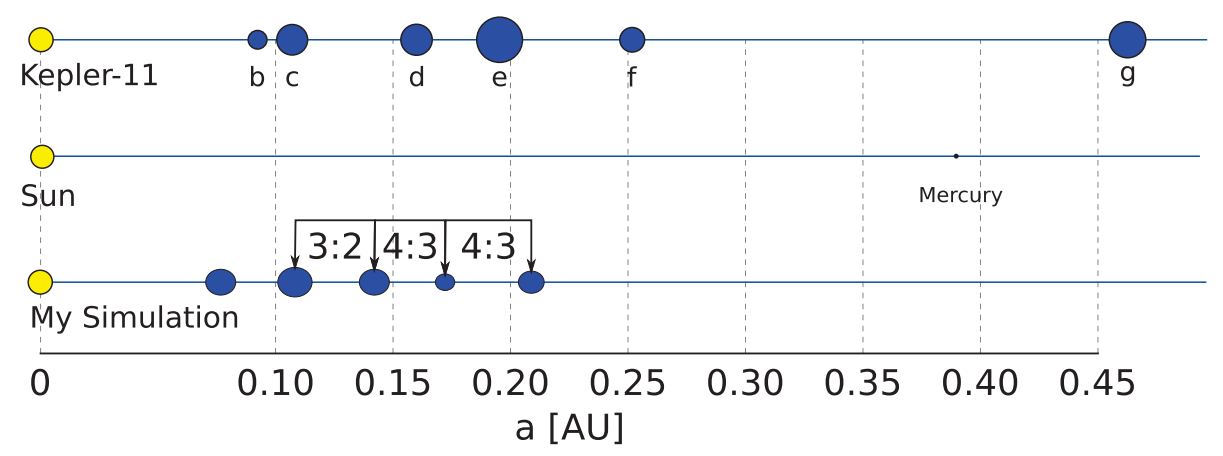

Figure 2. Final orbital configuration of the inner part of the simulation compared with the Solar System and Kepler 11.

position in the disk where the sum of all the torques felt by the planets cancels out (Cossou et al. 2013).

The left panel of Figure 1 shows the dynamics and accretion of a system of embryos embedded in the disk. The embryos comprised $60 \mathrm{M}_{\oplus}$ in total, and started with masses randomly chosen from $0.1-2 \mathrm{M}_{\oplus}$ spaced from $2-17 \mathrm{AU}$, and the integration lasted for 10 Myr. At early times, embryos migrate inward because their masses are below the $\sim 4 \mathrm{M}_{\oplus}$ threshold for outward migration (see right panel of Figure 1). Because of their different masses the embryos do not all migrate at the same rate. This leads to close encounters and collisions. Almost all of the embryos migrate inward and complete their growth close to the inner edge of the disk. The final configuration of planets is a compact resonant system. The most massive planet grows fast enough to reverse its migration and stabilizes at $15 \mathrm{AU}$. While migrating outward, it traps a lower mass planet in 6:5 resonance. The low-mass planet is pushed by the more massive one, and the two-planet system is effectively ruled by the zero-migration line of the more massive planet.

The embryos ended up at the inner edge of the disk for two reasons. First, objects less massive than $\sim 4 \mathrm{M}_{\oplus}$ simply cannot migrate outward (see Figure 1). During its inward migration, an embryo must accrete quickly if it is to enter a zone of outward migration, as was the case for the outer massive planet. Second, embryos that do migrate outward may encounter other large embryos and become trapped in resonance, leading to sustained 
non-zero eccentricities that damp the corotation torque (Bitsch \& Kley 2010) and tip the balance toward inward migration (Cossou et al. 2013). In this simulation the first mechanism dominated but both mechanisms can be important. The system was held up because a huge positive torque exists close to the inner edge due to the sudden decrease of the surface density profile (Masset et al. 2006). Our results are thus similar to those of Terquem \& Papaloizou (2007) even though outward migration does occur in our modeled disk.

Figure 2 shows the inner simulated system compared to our Solar System and the Kepler-11 system. The simulated system has similar masses to Kepler-11 but its orbital configuration is even more compact, although there are no planets in the simulation between 0.25 and $15 \mathrm{AU}$. The four outer simulated planets are in a resonant chain but the innermost planet is not. This is because the innermost planet was pushed into the disk's inner gas-free cavity where it lacked the energy dissipation required for efficient resonant trapping.

\section{Conclusion}

In our disk model, a compact system of hot Super Earths is formed by migration and accretion of planetary embryos. Inward migration is favored. Low-mass embryos naturally migrate inward. Outward migration is stalled or even reversed by the eccentricities sustained through resonances (Cossou et al. 2013). In essence, the presence of multiple large embryos leads to non-zero eccentricities such that they no longer follow the migration map (right panel of Figure 1).

This formation mechanism for hot Super Earths is robust against a much wider range of disk parameters than the one presented here. We note that the survival of the simulated hot Super Earths may depend on the detailed structure of the inner edge of the gas disk, which provides the positive torque needed to balance the inward-driven system. Their survival also likely depends on how the disk evolves (Horn et al. 2012) and especially on how it dissipates. We plan to take these effects, and others such as stochastic forcing from turbulence, into account in future work.

\section{References}

Bitsch, B. \& Kley, W. 2010, A\&BA, 523, A30

Chambers, J. E. 1999, MNRAS, 304, 793

Chiang, E. I. \& Goldreich, P. 1997, ApJ, 490, 368

Cossou, C., Raymond, S. N., \& Pierens, A. 2013, A\&A, 553, L2

Cresswell, P. \& Nelson, R. P. 2008, A\&̈A, 482, 677

Horn, B., Lyra, W., Mac Low, M.-M., \& Sándor, Z. 2012, ApJ, 750, 34

Howard, A. W., Marcy, G. W., Bryson, S. T., et al. 2012, ApJS, 201, 15

Huré, J.-M. 2000, A\&A, 358, 378

Lissauer, J. J., Fabrycky, D. C., Ford, E. B., et al. 2011, Nature, 470, 53

Masset, F. S., Morbidelli, A., Crida, A., \& Ferreira, J. 2006, ApJ, 642, 478

Mayor, M., Lovis, C., Pepe, F., Ségransan, D., \& Udry, S. 2011, Astronomische Nachrichten, 332,429

Menou, K. \& Goodman, J. 2004, ApJ, 606, 520

Paardekooper, S.-J., Baruteau, C., \& Kley, W. 2011, MNRAS, 410, 293

Shakura, N. I. \& Sunyaev, R. A. 1973, A\&A A, 24, 337

Terquem, C. \& Papaloizou, J. C. B. 2007, ApJ, 654, 1110 


\section{Discussion}

Schlichting: What determines the shape of the "whale"?

Cossou: The shape of the whale (regions of the migration map where the torque is positive) are mainly determined by the non-saturated entropy related part of the corotation torque, i.e a torque that is hugely sensitive on the temperature profile. Thus, any change in temperature will be seen directly in the migration map. But the shape itself comes from whether or not you have a non saturated corotation torque or not. Inside the "whale", the torque is not saturated, the migration is positive, and the following inequations are fullfilled $t_{\mathrm{U} \text {-turn }}<t_{\text {diff }}<t_{\text {libration. }}$. In the case of the whale, we always have $t$ visc $>t_{\text {rad }}$. The lower limit in mass of the whale is determined by the radiative diffusion timescale. When $t_{\mathrm{U} \text {-turn }}>t_{\mathrm{rad}}$, we are outside the whale because the corotation torque becomes linear. The upper mass limit of the whale comes when $t_{\mathrm{lib}} / 2>t_{\mathrm{visc}}$, thus the corotation torque saturates. But, the head of the whale does not exactly follow the equations $t_{\mathrm{U} \text {-turn }}=t_{\mathrm{rad}}$ and $t_{\mathrm{lib}} / 2=t_{\mathrm{visc}}$ because the commensurabilities between the main negative torque (Lindblad torque) and the main positive torque (horseshoe entropy related part of the corotation torque) changes due to transition from active to passive region of the disk. Past $4 \mathrm{AU}$, when the irradiation becomes dominant, Lindblad and corotation torques changes but not at the same rate. The final shape of the head of the whale is the line where the corotation torque (fully unsaturated or not) exactly balance the differential Lindblad torque. 